\title{
Extended annular capillary surfaces
}

\author{
Ray Treinen ${ }^{1}$
}

October 17, 2009

AMS subject classifications. 76B45, 49Q10, 35J60, 35J65.

\begin{abstract}
The height of the surface of a fluid in an annular tube is explored using a shooting method to solve a boundary value problem where the radii and the contact angles are given. The contact angles on the inner and outer tube surface need not be the same. These surfaces are then extended so that they are no longer graphs. The extended surfaces are shown to solve a boundary value problem over an annular base domain where given inclination angles are achieved at given radii.
\end{abstract}

\section{Introduction}

The equilibrium shape of the interface between liquid and air can be given in terms of the YoungLaplace equation

$$
\nabla \cdot\left(\frac{\nabla u}{\sqrt{1+|\nabla u|^{2}}}\right)=\kappa u
$$

where $\kappa=\rho g / \sigma$ is the capillary constant, where $\rho$ is the density of the fluid, $\sigma$ is the surface tension of the liquid surface, $g$ is the acceleration due to gravity. Solutions of this equation have been studied in a circular capillary tube, and the exterior of a cylinder, as well as more general domains. See Finn [4] for background and references.

Here we study the solution of a boundary value problem for (1) over an annular base domain assuming axial symmetry. This work extends results from Elcrat, Kim, and Treinen [1] where existence of the solution to the boundary value problem was given. In Section 2 short proofs of the main results in [1] are given using slightly different methods. In Sections 3 we extend the solution meridian, in the spirit of sessile drops, past vertical points to where they become horizontal.

In the remainder of this section some equivalent forms of (1) are given and the different boundary conditions are explained and grouped into different problems.

Under the assumption of symmetry (1) becomes

(2) $\left(\frac{r u^{\prime}(r)}{\sqrt{1+\left(u^{\prime}(r)\right)^{2}}}\right)^{\prime}=\kappa r u(r)$

where $r$ is the radial coordinate and $u=u(r)$ is the height of the surface. Here and in what follows $(\cdot)^{\prime}$ denotes differentiation with respect to $r$, even if further parameters are introduced.

It is convenient to introduce the inclination angle $\psi$, measured from the positive direction of the horizontal component. Then (2) may be written more compactly:

(3) $\quad(r \sin \psi)^{\prime}=\kappa r u$.

\footnotetext{
${ }^{1}$ Department of Mathematics, Kansas State University, Manhattan, KS 66502
} 
This then leads to a system of first order equations

$$
\text { (4) } \begin{aligned}
\frac{d r}{d \psi} & =\frac{r \cos \psi}{\kappa r u-\sin \psi} \\
\text { (5) } \frac{d u}{d \psi} & =\frac{r \sin \psi}{\kappa r u-\sin \psi},
\end{aligned}
$$

which hold on any portion of the solution curve that does not contain an inflection point. One further form of the differential equations, parametrized by arc length $s$, is:

$\begin{aligned} \text { (6) } \frac{d r}{d s} & =\cos \psi \\ \text { (7) } \frac{d u}{d s} & =\sin \psi \\ \text { (8) } \frac{d \psi}{d s} & =\kappa u-\frac{\sin \psi}{r} .\end{aligned}$

Consider a container bounded by two concentric circular cylindrical walls. The base domain is a circular annulus. We prescribe the inner radius $a$ and the outer radius $b$ as well as the contact angles at each: $\gamma_{a}, \gamma_{b}$. Note that the contact angles are measured within the fluid, so that $0<\gamma_{a}<\pi / 2$ corresponds to a negative slope for the tangent at $a$ and $0<\gamma_{b}<\pi / 2$ corresponds to a positive slope for the tangent at $b$. The given contact angles then lead to given inclination angles $\psi_{a}$ and $\psi_{b}$ where the sign of the inclination angle is the same as the sign of the slope of the tangent.

We thus arrive at the problem considered in [1] which consists of finding a solution $u \in C^{1}[a, b]$ of (1) with prescribed boundary inclination angles $\psi_{a}, \psi_{b} \in(-\pi / 2, \pi / 2)$. All solutions obtained in [1] are single valued with respect to the radial variable and naturally have inclination angle satisfying $\psi \in(-\pi / 2, \pi / 2)$.

The equations (4),(5) admit parametric solutions with inclination angle taking values both inside and outside the interval $(-\pi / 2, \pi / 2)$. This makes it natural to consider an analogous problem for (4),(5) with more general boundary conditions. The natural extension would be to include inclination angles $\psi_{a}, \psi_{b} \in[-\pi, \pi]$. This leads to the possibility of an inflection point on the curve, in which case (6). (7), (8) are needed.

It will be observed that the projection of the solution profile projects neither simply onto the radial axis, nor onto the interval $[a, b]$. We call any solution of (6). (7), (8) with these properties the profile of an extended capillary surface.

These profiles include several capillary interfaces of interest, as indicated in Figure 1, Figure 2 and Figure 3. Figure 1 shows the profile of sessile drop of a non-wetting liquid satisfying $\psi_{a} \in(-\pi / 2,0$ and $\psi_{b} \in[-\pi,-\pi / 2)$. In Figure 2 a sphere floats in a container partially filled with fluid. The dashed lines appear at $r= \pm 0.7$. Set $a=0.7$ and $b=2$. The fluid interface is an extended capillary surface, with $\psi_{a} \in[-\pi, \pi]$ and $\psi_{b} \in[-\pi / 2, \pi / 2]$. In Figure 3 a drop of fluid floats in a bath of another fluid. The container is an annular cylinder, with walls at radii $r=0.1$ and $r=0.5$. The dashed lines appear at the triple junction $\bar{r}=0.25$. There are three extended capillary surfaces: two with $a=0.1$ and $b=0.25$, where $\psi_{a} \in[-\pi / 2, \pi / 2]$ and $\psi_{b} \in[-\pi, \pi]$, and the third with $a=0.25$ and $b=0.5$, where $\psi_{a} \in[-\pi, \pi]$ and $\psi_{b} \in[-\pi / 2, \pi / 2]$. In Section 3 we cover the case $\psi_{a} \in[-\pi, 0]$ and $\psi_{b} \in[0, \pi]$.

Additional discussion of floating drops and applications of extended capillary surfaces may be found in [2], [3], and [8]. A discussion of a floating sphere in a bounded container may be found in [6]. 


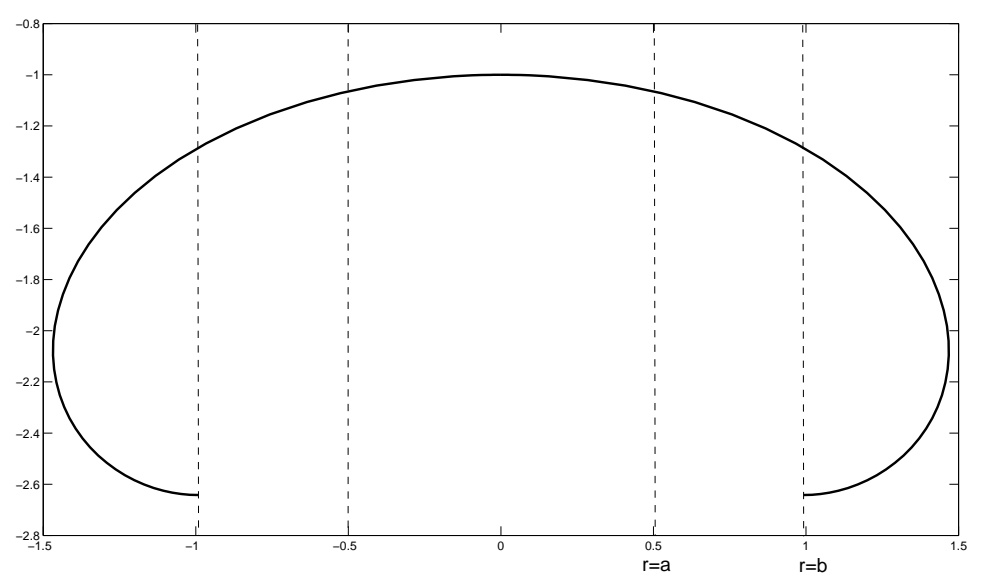

Figure 1: Vertical section of sessile drop, passing through two annular walls.

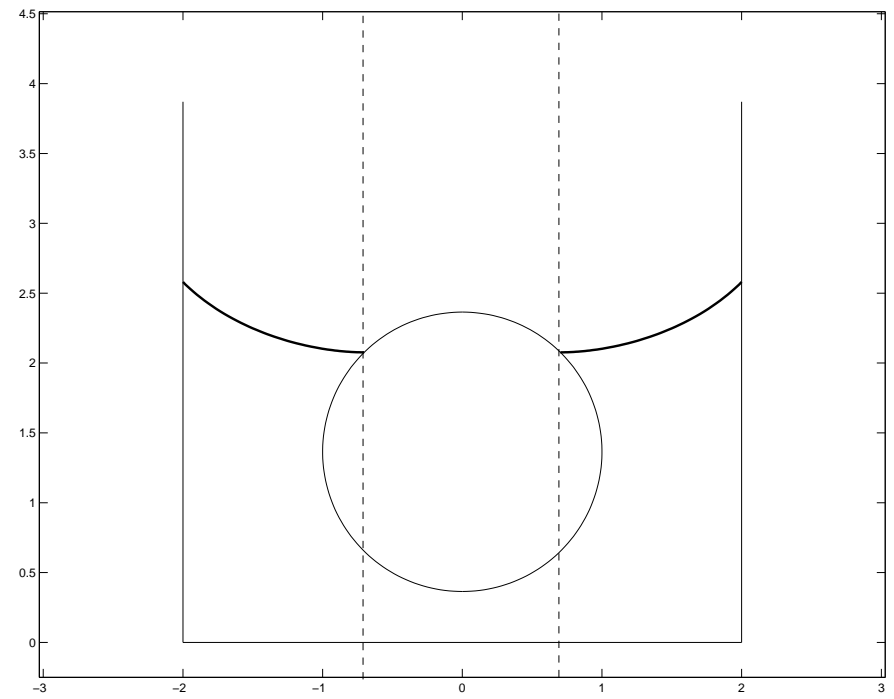

Figure 2: Vertical section of a sphere floating on a liquid. 


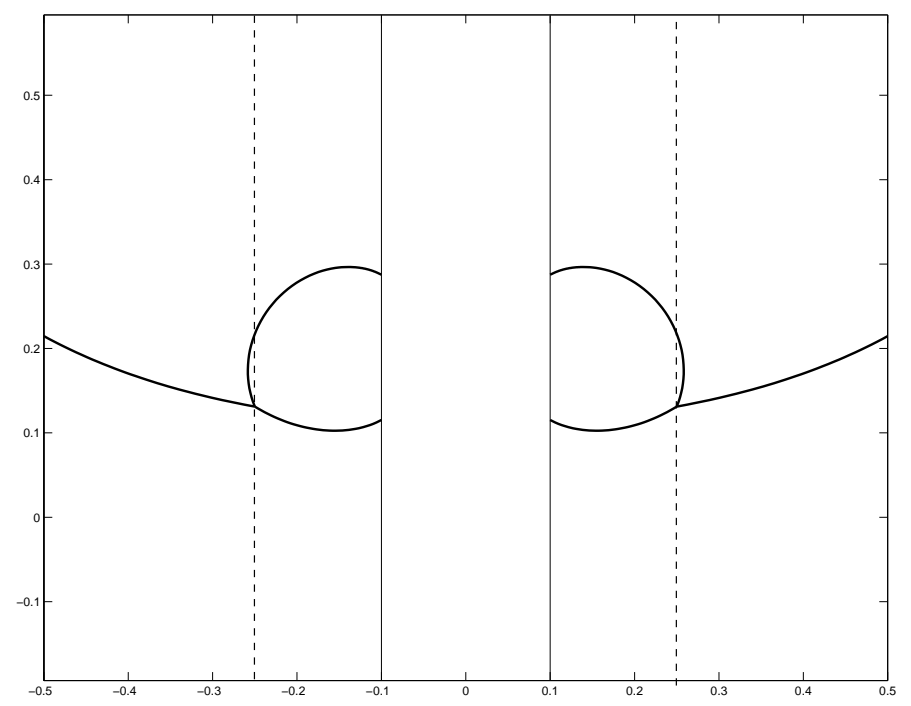

Figure 3: Vertical section of an axial symmetric floating drop.

\section{Solutions that are graphs}

Consider the differential equation (2) with boundary conditions $\psi_{a}, \psi_{b} \in(-\pi / 2, \pi / 2)$ with $\psi_{a}$ prescribed at $a$ and $\psi_{b}$ prescribed at $b$. In proving existence of the solution to this boundary value problem we first derive integral equations. We will use a shooting method, so we consider first the left boundary point $a$ with $\psi_{a}$ given and fixed. Integrating (2) from $a$ to $r$ for $a<r \leq b$ and using $(3)$,

(9) $\frac{u^{\prime}(r)}{\sqrt{1+u^{\prime}(r)^{2}}}=\frac{a}{r} \sin \psi_{a}+\frac{\kappa}{r} \int_{a}^{r} u(\xi) \xi d \xi$.

Set

(10) $v(r)=\frac{a}{r} \sin \psi_{a}+\frac{\kappa}{r} \int_{a}^{r} u(\xi) \xi d \xi$

and solving for $u^{\prime}(r)$, then integrating from $a$ to $r$, while for convenience, denoting $h=u(a)$ :

(11) $u(r)=h+\int_{a}^{r} \frac{v(\xi)}{\sqrt{1-v(\xi)^{2}}} d \xi$.

Note that $h$ is a parameter here: as convenient we will write $u(r ; h)$ and $v(r ; h)$. The plan is to use integral equations (10) and (11) to show that we may attain any admissible boundary values by considering a range of values for $h$.

It will be useful to consider the unbounded liquid bridge, which is the solution the the problem

(12) $\begin{cases}(r \sin \psi)^{\prime}=\kappa r w & \\ \cos \gamma=\sin \psi_{a} & \text { at } a \\ w=0 & \text { at } \infty .\end{cases}$ 
This problem has been studied extensively, see Johnson and Perko [5], Siegel [7], Turkington [9], and Vogel [10]. The first results are when the solution is a graph, and Vogel's results extend this to functions that are not graphs. Vogel's results can be slightly modified so that (12) is equivalent to (4) and (5) with $\psi_{a}=-\pi / 2$ at $r=a$ and $w(a)=T(a)$ where the function $T$ is the unique height of the vertical point $(\sigma, T(\sigma))$ so that $w=0$ at $\infty$ (this corresponds to $\psi=0$ at $\infty$ ). Elcrat, Neel, and Siegel [2] proved there is a unique liquid bridge for any given $\psi_{a}$ with $0 \leq \psi_{a}<\pi / 2$. Thus there is a surface $w$ that satisfies the left boundary condition, the same equivalent differential equations, and extends to any radius.

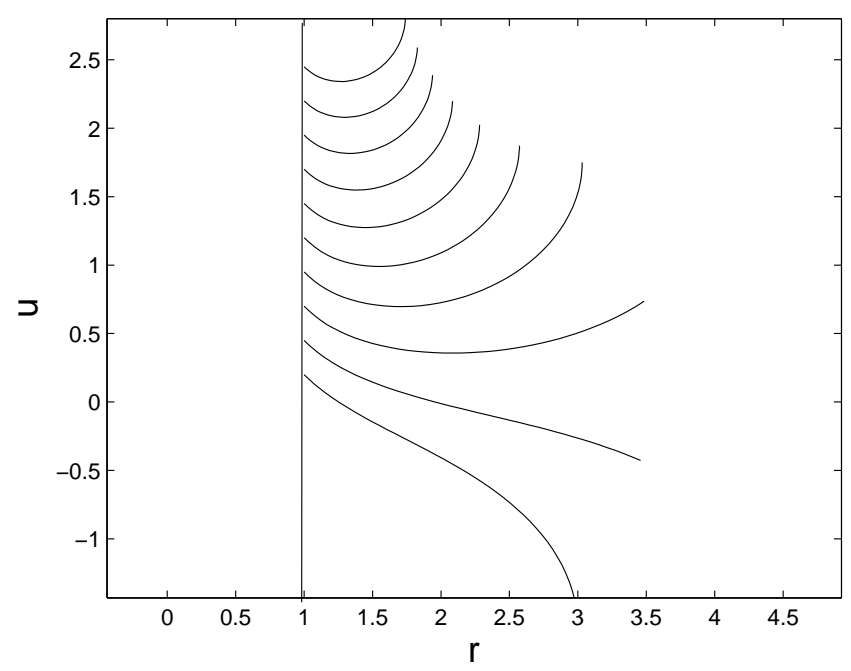

Figure 4: Sweeping through a family of solution curves.

The following lemmas are slightly modified from [1]. This process is summarized in Figure 4.

Lemma 2.1 For $h_{1}<h_{2}$ and $a<r \leq b$ we have $u\left(r ; h_{1}\right)<u\left(r ; h_{2}\right)$ and $u^{\prime}\left(r ; h_{1}\right)<u^{\prime}\left(r ; h_{2}\right)$.

Proof. Note that $u^{\prime}\left(a ; h_{1}\right)=u^{\prime}\left(a ; h_{2}\right)$ as $\psi_{a}$ is given, and the same for both $u\left(r ; h_{1}\right)$ and $u\left(r ; h_{2}\right)$. Also note that $f(p):=p / \sqrt{1-p^{2}}$ is an increasing function. Then, for some $\epsilon>0$ and $a \leq r \leq a+\epsilon$, as $u$ is continuous and $h_{1}<h_{2}$

$$
\int_{a}^{r} u\left(\xi ; h_{1}\right) \xi d \xi<\int_{a}^{r} u\left(\xi ; h_{2}\right) \xi d \xi
$$

Then from (9) and the monotonicity of $f(p)$ we find that $u^{\prime}\left(r ; h_{1}\right)<u^{\prime}\left(r ; h_{2}\right)$ on $a<r \leq a+\epsilon$. Thus $u\left(r ; h_{1}\right)<u\left(r ; h_{2}\right)$ on $(a, a+\epsilon)$.

If the conclusion of the lemma fails at some first $r_{0}>a$, then it must be the case that $u\left(r_{0} ; h_{1}\right) \leq$ $u\left(r_{0} ; h_{2}\right)$ and $u^{\prime}\left(r_{0} ; h_{1}\right)=u^{\prime}\left(r_{0} ; h_{2}\right)$ with

(14) $u^{\prime \prime}\left(r_{0} ; h_{1}\right)>u^{\prime \prime}\left(r_{0} ; h_{2}\right)$.

We are, however, in the same situation as we were at $r=a$, and by replacing $a$ with $r_{0}$ and repeating the argument above, we see that $u^{\prime}\left(r ; h_{1}\right)<u^{\prime}\left(r ; h_{2}\right)$ on $\left(r_{0}, r_{0}+\epsilon\right)$. Differentiating (9) and evaluating at $r_{0}$, we find

(15) $\left[f\left(u^{\prime}\left(r_{0} ; h_{1}\right)\right)\right]^{\prime}<\left[f\left(u^{\prime}\left(r_{0} ; h_{2}\right)\right)\right]^{\prime}$.

Since $f(p)$ has a positive derivative, this implies $u^{\prime \prime}\left(r_{0} ; h_{1}\right)<u^{\prime \prime}\left(r_{0} ; h_{2}\right)$, contradicting (14). 
Lemma 2.2 There exist $h_{1}$ and $h_{2}$, where $h_{1}<h_{2}$, such that if $h_{1}<h<h_{2}$ then $|u(r ; h)|,\left|u^{\prime}(r ; h)\right|<$ $\infty$ on $[a, b]$.

Proof. First take $h_{0}$ to be the height of the unbounded liquid bridge that has the inclination angle $\psi_{a}$ at $r=a$. From continuous dependence on the data $h$, we may find $h_{2}>h_{0}$ as follows: given $\epsilon>0$, for $\delta>0$ sufficiently small we have $u\left(b ; h_{0}+\delta\right)<u\left(b ; h_{0}\right)+\epsilon$ and $u^{\prime}\left(b ; h_{0}+\delta\right)<u^{\prime}\left(b ; h_{0}\right)+\epsilon$. Set $h_{2}=h_{0}+\delta$. By Lemma 2.1 we may assert the claim for any $h \in\left[h_{0}, h_{2}\right]$. If the height is decreased from $h_{0}$ we obtain $u\left(b ; h_{0}-\delta\right)>u\left(b ; h_{0}\right)-\epsilon$ and $u\left(b ; h_{0}-\delta\right)>u\left(b ; h_{0}\right)-\epsilon$ and if we take $h_{1}=h_{0}-\delta$ we may again assert our claim for any $h \in\left[h_{1}, h_{0}\right]$.

Lemma $2.3 \max _{h_{2}} u^{\prime}\left(b ; h_{2}\right)=\infty$ and $\min _{h_{1}} u^{\prime}\left(b ; h_{1}\right)=-\infty$.

Proof. Considering Lemma 2.1, either $\sup u^{\prime}\left(b ; h_{2}\right)=\infty$ or $\sup u^{\prime}\left(b ; h_{2}\right)<\infty$. Denote the value of $h$ (in $\overline{\mathbb{R}}$ ) that attains this supremum by $\tilde{h}_{2}$. If $\tilde{h}_{2}=\infty$ then $u\left(a ; \tilde{h}_{2}\right)=\infty$. This implies $u^{\prime}\left(r ; \tilde{h}_{2}\right)=\infty$ before $r=b$ and as (9) implies $u^{\prime}(r ; h)$ is increasing in $r(h>0)$. Thus $\tilde{h}_{2}<\infty$ as $u^{\prime}(r ; h)$ is increasing in $h$, and therefore the supremum is achieved in $\mathbb{R}$.

Assume $\sup u^{\prime}\left(b ; h_{2}\right)<\infty$. Take $\tilde{h}_{2}+\epsilon$ for $\epsilon>0$ small enough that $u^{\prime}\left(b ; \tilde{h}_{2}+\epsilon\right)<\infty$, which is possible by continuity. Then Lemma 2.1 implies $u^{\prime}\left(b ; \tilde{h}_{2}\right)<u^{\prime}\left(b ; \tilde{h}_{2}+\epsilon\right)$, and this contradicts that $\tilde{h}_{2}$ was the max.

The proof for $\min u^{\prime}\left(b ; h_{1}\right)=-\infty$ is similar.

Set $\tilde{h}_{1}$ as value for $h_{1}$ achieving the infimum above. Now we see that $\left\{u^{\prime}(b ; h) \mid \tilde{h}_{1} \leq h \leq \tilde{h}_{2}\right\} \supseteq$ $(-\infty, \infty)$ and this proves the following theorem:

Theorem 2.4 Let $\psi_{a}, \psi_{b} \in(-\pi / 2, \pi / 2)$. There exists a solution to equation (2) with boundary conditions $\psi_{a}$ prescribed at $a$ and $\psi_{b}$ prescribed at $b$.

\section{$3 \quad$ Inclination angles $\psi_{a} \in[-\pi, 0]$ and $\psi_{b} \in[0, \pi]$}

Many of the ideas in what follows are variations of methods used in Finn [4], Chapter 3, with sessile drops. We begin with a point $(m, h)$ such that $u(m)=h>0$ and $u^{\prime}(m)=0$. Integrating (3) from $m$ to $r>m$, then solving for $k_{l}:=\sin \psi / r$, and using the monotonicity of $u(r)$ yields:

(16) $\frac{\kappa u_{m}}{2}\left(1-\frac{m^{2}}{r^{2}}\right)<\frac{\sin \psi}{r}<\frac{\kappa u}{2}\left(1-\frac{m^{2}}{r^{2}}\right)$.

A similar estimation produces

(17) $\frac{\kappa u_{m}}{2}\left(\frac{m^{2}}{r^{2}}-1\right)<\frac{-\sin \psi}{r}<\frac{\kappa u}{2}\left(\frac{m^{2}}{r^{2}}-1\right)$.

to the left of $m$. These estimates hold up to vertical points.

For $\psi \in(0, \pi / 2),(16)$ implies

(18) $2 \sin \psi<\kappa r u\left(1-\frac{m^{2}}{r^{2}}\right)=\kappa r u-\frac{\kappa u m^{2}}{r}$

which implies

(19) $0<\sin \psi<\frac{\kappa u m^{2}}{r}+\sin \psi<\kappa r u-\sin \psi$.

This implies that there are no singularities to (4),(5) at $\psi=\pi / 2$. Denote $R:=r(\pi / 2)$. As $\psi<0$ to the left of $m$ and $r, u>0$ there are no singularities at the left vertical point $L:=r(-\pi / 2)$.

The left inequality of (16) with $\psi=\pi / 2$ implies a bound on $R$ :

$$
\begin{aligned}
\frac{\kappa u_{m}}{2}\left(1-\frac{m^{2}}{R^{2}}\right) & <\frac{1}{R} \\
\frac{\kappa u_{m}}{2} R^{2}-R-\frac{\kappa u_{m}}{2} m^{2} & <0,
\end{aligned}
$$


then

(20) $\quad R<\frac{1+\sqrt{1+\kappa^{2} u_{m}^{2} m^{2}}}{\kappa u_{m}}<\infty$.

Similarly using the left half of (17) we obtain

(21) $L>\frac{-1+\sqrt{1+\kappa^{2} u_{m}^{2} m^{2}}}{\kappa u_{m}}>0$.

Note as $u_{m} \rightarrow \infty$ these estimates imply $L, R \rightarrow m$. In fact, in [1] it was shown that $u_{m} \rightarrow 0$ implies $L \rightarrow 0$ and $R \rightarrow \infty$. The simplified methods used in this current paper do not make this fact obvious.

The object is to continue the solution meridian to have inclination angle $\pi$ on the right and to have inclination angle $-\pi$ on the left. Consider the right part of the meridian first. With (4),(5) it is possible to continue past $R$ to some $\psi \in(\pi / 2, \pi]$. On this part of the meridian (3) continues to hold. Label the part of the meridian where $\psi>\pi / 2$ by $(+)$ and the part where $\psi<\pi / 2$ by $(-)$. Label the left part of the meridian similarly. See Figure 5.

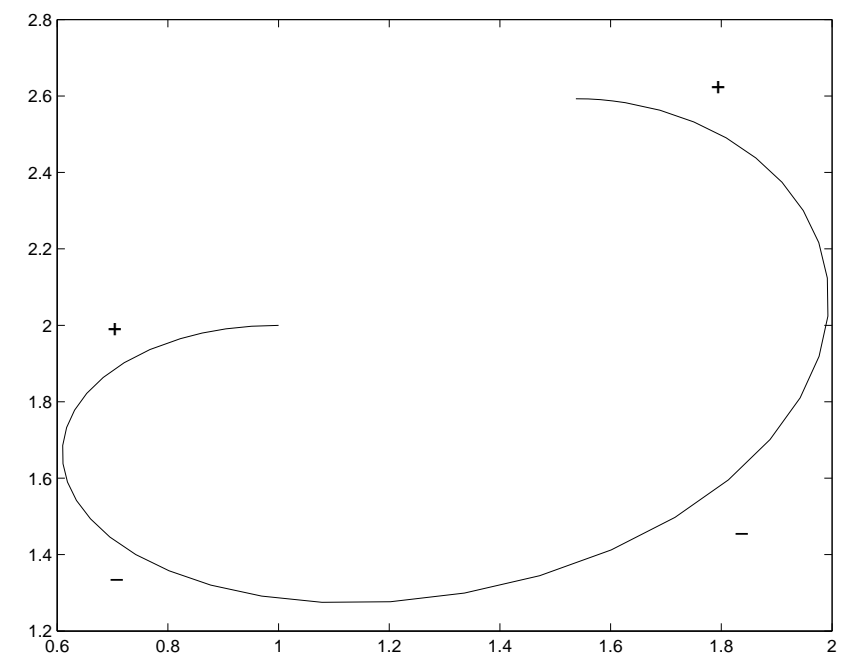

Figure 5: The regions $(+)$ and $(-)$ on the left and right.

Integrating from $r$ to $R$ on both (+) and (-) and subtracting:

(22) $r\left(\sin \psi^{-}-\sin \psi^{+}\right)=\kappa \int_{r}^{R}\left(u^{+}-u^{-}\right) \rho d \rho$.

By (5) this implies $u^{+}>u^{-}$for $r$ near $R$, and thus $\sin \psi^{-}>\sin \psi^{+}$. This implies (19) holds as long as continuation is valid. As $u^{+}$and $u^{-}$are increasing in $\psi$, then $\sin \psi^{-}>\sin \psi^{+}$and thus $u^{+}>u^{-}$ as long as possible. If $r \rightarrow m$ then continuation would be possible up to $m$. As continuation stops with $\psi=\pi$ and $\sin \psi^{-}>\sin \psi^{+}$continuation must stop before $m$.

For $\psi \in[-\pi / 2,0]$ we first note that $\kappa r u-\sin \psi>0$, as it is proven in [1] that $L>0$. Then as $\sin \psi<0, \frac{d r}{d \psi}>0$ and $\frac{d u}{d \psi}<0$. Continuing past $\psi=\pi / 2, \kappa r u-\sin \psi^{+}>0$ and thus $\frac{d r}{d \psi}<0$ and $\frac{d u}{d \psi}<0$ for $\psi \in[-\pi,-\pi / 2]$. Then (3) still holds for $\psi \in(-\pi,-\pi / 2)$, so integrating from $L$ to $r$ on $(-)$ and $(+)$ and subtracting:

(23) $r\left(\sin \left(-\psi^{-}\right)-\sin \left(-\psi^{+}\right)\right)=\kappa \int_{L}^{r}\left(u^{+}-u^{-}\right) \rho d \rho$.

Then $\sin \psi^{-}<\sin \psi^{+}$and $u^{+}>u^{-}$as long as continuation is possible, as in the other case. Then as $r \rightarrow m$ we have $\sin \psi^{-} \rightarrow 0$ from below and continuation breaks down before $r=m$. 
Lemma 3.1 For the meridian determined by $u\left(\psi ; u_{m}, m\right), k_{l}$ is increasing on $(-\pi / 2, \pi / 2)$ and decreasing on $(-\pi,-\pi / 2)$ and $(\pi / 2, \pi)$.

Proof. Calculating

$$
\begin{aligned}
\left(k_{l}\right)_{\psi} & =\left(\frac{\sin \psi}{r}\right)_{\psi} \\
& =\frac{\cos \psi}{r}-\frac{\sin \psi}{r^{2}} \frac{d r}{d \psi} \\
& =\frac{\cos \psi}{r}-\frac{\sin \psi}{r^{2}} \frac{r \cos \psi}{\kappa r u-\sin \psi} \\
& =\frac{\cos \psi}{r}\left(1-\frac{\sin \psi}{\kappa r u-\sin \psi}\right) \\
& =\frac{\cos \psi}{r} \frac{\kappa r u-2 \sin \psi}{\kappa r u-\sin \psi} .
\end{aligned}
$$

Observe that (19) holds on the entire interval $0<\psi<\pi$ :

$$
\frac{\kappa r u-2 \sin \psi}{\kappa r u-\sin \psi}>\frac{\kappa r u-\sin \psi}{\kappa r u-\sin \psi}=0
$$

Also note that

$$
\frac{\kappa r u-2 \sin \psi}{\kappa r u-\sin \psi}>0
$$

on $-\pi<\psi<0$. The result follows from the $\operatorname{sign}$ of $\cos \psi$ on the interval $(-\pi, \pi)$.

Note that this implies that $\sin \psi / r$ attains a minimum of $-1 / L$ when $r=L$, and a maximum of $1 / R$ when $r=R$.

Lemma 3.2 On $-\pi \leq \psi \leq-\pi / 2$ there holds

(25) $u(\psi)<\frac{\sin \psi}{\kappa r}+\sqrt{\frac{-2}{\kappa} \cos \psi+\left(u_{L}-\frac{\sin \psi}{\kappa r}\right)^{2}}$

and

(26) $u(\psi)>\frac{-1}{\kappa L}+\sqrt{\frac{-2}{\kappa} \cos \psi+\left(u_{L}+\frac{1}{\kappa L}\right)^{2}}$

and on $\pi / 2 \leq \psi \leq \pi$ there holds

(27) $u(\psi)>\frac{\sin \psi}{\kappa r}+\sqrt{\frac{-2}{\kappa} \cos \psi+\left(u_{R}-\frac{\sin \psi}{\kappa r}\right)^{2}}$

and

(28) $u(\psi)<\frac{1}{\kappa R}+\sqrt{\frac{-2}{\kappa} \cos \psi+\left(u_{R}-\frac{1}{\kappa R}\right)^{2}}$.

Proof. Rearranging (5) then integrating from $\pi / 2$ to $\psi$ :

$$
\begin{aligned}
\int_{u_{R}}^{u}\left(u-\frac{\sin \psi}{\kappa r}\right) d u & =\int_{\pi / 2}^{\psi} \frac{\sin \psi}{\kappa} d \psi \\
\text { (29) } \frac{1}{2}\left(u^{2}-u_{R}^{2}\right)-\int_{u_{R}}^{u} \frac{\sin \psi}{\kappa r} d u & =\frac{-\cos \psi}{\kappa} .
\end{aligned}
$$


Now $k_{l}$ decreases over the range of integration (note $d \psi / d s>0$ on the entire length of the meridian), so as the smallest value is $\sin \psi / r$, the left side of the last equation is increased:

(30) $\frac{\sin \psi}{\kappa r}+\sqrt{\frac{-2 \cos \psi}{\kappa}+\left(u_{R}-\frac{\sin \psi}{\kappa r}\right)^{2}}<u(\psi)$.

Now continuing from (29) we estimate $k_{l}$ by its largest value of $1 / R$, the left side of $(29)$ is decreased:

$$
\begin{aligned}
& \frac{1}{2}\left(u^{2}-u_{R}^{2}\right)-\frac{1}{\kappa R}\left(u-u_{R}\right)<\frac{-\cos \psi}{\kappa} \\
&\left(u-\frac{1}{\kappa R}\right)^{2}-\left(u_{R}-\frac{1}{\kappa R}\right)^{2}<\frac{-2 \cos \psi}{\kappa} \\
& \text { (31) } \frac{1}{\kappa R}+\sqrt{\frac{-2 \cos \psi}{\kappa}+\left(u_{R}-\frac{1}{\kappa R}\right)^{2}}>u(\psi)
\end{aligned}
$$

as above. Similarly on the left part of the solution meridian, integrating from $\psi$ to $-\pi / 2$ we have

$$
\begin{aligned}
\int_{u_{L}}^{u}\left(u-\frac{\sin \psi}{\kappa r}\right) d u & =\int_{-\pi / 2}^{\psi} \frac{\sin \psi}{\kappa} d \psi \\
\text { (32) } u^{2}-u_{L}^{2}-\int_{u_{L}}^{u} \frac{2 \sin \psi}{\kappa r} d u & =-\frac{2 \cos \psi}{\kappa} .
\end{aligned}
$$

Then by Lemma 3.1 we have

$$
\begin{aligned}
u^{2}-u_{L}^{2}+\frac{2}{\kappa L}\left(u-u_{L}\right) & >-\frac{2 \cos \psi}{\kappa} \\
\frac{-1}{\kappa L}+\sqrt{\frac{-2 \cos \psi}{\kappa}+\left(u_{L}+\frac{1}{\kappa L}\right)^{2}} & <u(\psi)
\end{aligned}
$$

and

(34) $\frac{\sin \psi}{\kappa r}+\sqrt{\frac{-2 \cos \psi}{\kappa}+\left(u_{L}-\frac{\sin \psi}{\kappa r}\right)^{2}}>u(\psi)$.

Before proving the next lemma we make a remark.

Remark 3.3 It would seem natural to seek $\alpha:=r(-\pi) \rightarrow 0$ as $h:=u_{m} \rightarrow 0$, but this appears not to be true in general, as numerical experiments have suggested: see Figure 6.

Lemma 3.4 For the meridian determined by $u\left(\psi ; u_{m}, m\right), \beta:=r(\pi) \rightarrow \infty$ as $h:=u_{m} \rightarrow 0$, and $\alpha, \beta \rightarrow m$ as $h \rightarrow \infty$. Furthermore, $h \rightarrow 0$ implies that if $\pi / 2<\psi \leq \pi$, then $r(\psi, h, m) \rightarrow \infty$ uniformly.

Proof. It has already been noted that as $h \rightarrow \infty$ that $L, R \rightarrow m$. Thus as $L<\alpha<m<\beta<R$ we have $\alpha, \beta \rightarrow m$ as $h \rightarrow \infty$.

In [1] it was proven that as $h \rightarrow 0$ that $L \rightarrow 0$ and $R \rightarrow \infty$. Using that as a starting place we show $R \rightarrow \infty$ implies $\beta \rightarrow \infty$ with the following calculation: rearranging (4) and integrating from 


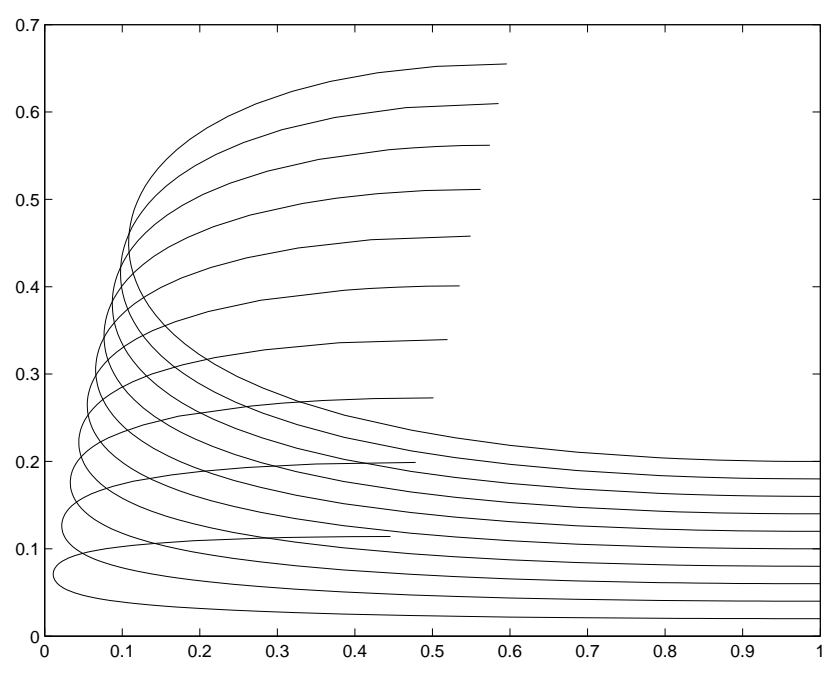

Figure 6: We decrease the height $h$ at $m=1, \alpha$ decreases but we do not have $\alpha \rightarrow 0$.

$\pi / 2$ to $\psi$, then estimating with (28) and using Lemma 3.1 gives

$$
\begin{aligned}
\int_{R}^{r}\left(u-\frac{\sin \psi}{\kappa r}\right) d r & =\int_{\pi / 2}^{\psi} \frac{\cos \psi}{\kappa} d \psi \\
\int_{R}^{r}\left(\frac{1}{\kappa R}+\sqrt{\frac{-2}{\kappa} \cos \psi+\left(u_{R}-\frac{1}{\kappa R}\right)^{2}}-\frac{\sin \psi}{\kappa r}\right) d r & >\frac{\sin \psi}{\kappa}-\frac{1}{\kappa} \\
\left(\frac{1}{\kappa R}+\sqrt{\frac{-2}{\kappa} \cos \psi+\left(u_{R}-\frac{1}{\kappa R}\right)^{2}}-\frac{\sin \psi}{\kappa r}\right)(r-R) & >\frac{\sin \psi}{\kappa}-\frac{1}{\kappa} \\
\frac{r}{\kappa R}+\frac{R}{r} \frac{\sin \psi}{\kappa}+(r-R) \sqrt{\frac{-2}{\kappa} \cos \psi+\left(u_{R}-\frac{1}{\kappa R}\right)^{2}} & >\frac{2 \sin \psi}{\kappa} .
\end{aligned}
$$

Now, putting $\psi=\pi$ gives

(36) $\frac{\beta}{\kappa R}+(\beta-R) \sqrt{\frac{2}{\kappa}+\left(u_{R}-\frac{1}{\kappa R}\right)^{2}}>0$,

and taking the limit $R \rightarrow \infty$ implies that $\beta \rightarrow \infty$ as well. That $\beta \leq r(\psi, h, m)$ for $\pi / 2<\psi \leq \pi$ implies the uniform convergence in that range of $\psi$.

Define the volumes $V_{L}$ and $V_{R}$ to be the volumes generated by the areas between the curve $(r, u)$, the line $r=m$, and the lines $u=u\left(\psi_{a}\right)$ and $u=u\left(\psi_{b}\right)$ respectively. Denote $V=V_{L}+V_{R}$. See Figure 7. Denote $\Delta=\kappa r u-\sin \psi$. We compute

(37) $\quad V_{L}=\pi\left(m^{2}-r^{2}\right) u+\frac{2 \pi}{\kappa} r \sin \psi$

(38) $V_{R}=\pi\left(r^{2}-m^{2}\right) u-\frac{2 \pi}{\kappa} r \sin \psi$.

It can be seen from the standard continuous dependence theorems that $V$ is a continuously differentiable function of $u_{m}$. Thus, we may define

(39) $\quad \dot{V}=\dot{V}_{L}+\dot{V}_{R}:=\frac{\partial V_{L}\left(\psi ; u_{m}, m\right)}{\partial u_{m}}+\frac{\partial V_{R}\left(\psi ; u_{m}, m\right)}{\partial u_{m}}$ 


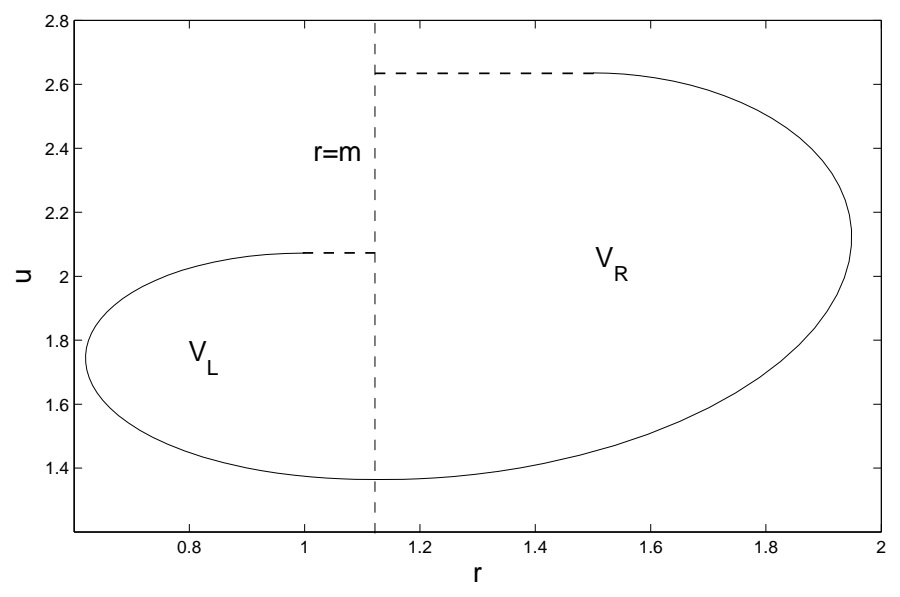

Figure 7: Generating curves for the volumes $V_{L}$ and $V_{R}$.

and $\dot{r}:=\partial r / \partial u_{m}$ and $\dot{u}:=\partial u / \partial u_{m}$. Then

(40) $\dot{V}_{L}=\pi\left[-\frac{2 \dot{r}}{\kappa} \Delta+\left(m^{2}-r^{2}\right) \dot{u}\right]$,

(41) $\dot{V}_{R}=\pi\left[\frac{2 \dot{r}}{\kappa} \Delta+\left(r^{2}-m^{2}\right) \dot{u}\right]$.

We calculate

(42) $\frac{d \dot{V}_{L}}{d \psi}=\pi \frac{r^{2} \sin \psi}{\Delta^{2}}\left\{\dot{r}\left[-2 \Delta-\frac{m^{2}-r^{2}}{r^{2}} \sin \psi\right]-\kappa\left(m^{2}-r^{2}\right) \dot{u}\right\}$,

(43) $\frac{d \dot{V}_{R}}{d \psi}=\pi \frac{r^{2} \sin \psi}{\Delta^{2}}\left\{\dot{r}\left[2 \Delta-\frac{r^{2}-m^{2}}{r^{2}} \sin \psi\right]-\kappa\left(r^{2}-m^{2}\right) \dot{u}\right\}$.

and also

(44) $\frac{d \dot{r}}{d \psi}=-\frac{\kappa r^{2} \dot{u}+\dot{r} \sin \psi}{\Delta^{2}} \cos \psi$

(45) $\frac{d \dot{u}}{d \psi}=-\frac{\kappa r^{2} \dot{u}+\dot{r} \sin \psi}{\Delta^{2}} \sin \psi$

with

(46) $\dot{r}(0)=0, \quad \dot{u}(0)=1$.

For $L<r<R$ we have

(47) $r \sin \psi=\kappa \int_{m}^{r} \rho u d \rho$.

and writing $u=u\left(\rho ; u_{m}, m\right)=u\left(\rho\left(\psi ; u_{m}, m\right) ; u_{m}, m\right)$,

(48) $\left.\left.\left.\left.\frac{\partial u}{\partial u_{m}}\right]_{\psi}=\frac{\partial u}{\partial \rho}\right]_{\left(u_{m}, m\right)} \dot{\rho}+\frac{\partial u}{\partial u_{m}}\right]_{\rho}=\dot{\rho} \tan \psi+\frac{\partial u}{\partial u_{m}}\right]_{\rho}$

Using (47) we calculate

(49) $-\dot{r} \Delta=\kappa \int_{m}^{r}(\dot{u}-\dot{\rho} \tan \psi) \rho d \rho$. 
Using (46) we see that for each $u_{m}>0$ there is an interval $\mathcal{I}_{R}: 0<\psi<\delta \leq \pi$, in which $\dot{r}<0$, and also an interval $\mathcal{I}_{L}: 0>\psi>\delta \geq-\pi$, in which $\dot{r}>0$. In both $\mathcal{I}_{L}$ and $\mathcal{I}_{R}$, by (45),

(50) $\frac{d \dot{u}}{d \psi}>-\frac{\kappa r^{2} \dot{u} \sin \psi}{\Delta^{2}}$

as in both cases $\dot{r} \sin \psi>0$. Note $\Delta=\Delta(\psi)=\kappa r u-\sin \psi \neq 0$ is a continuous function of $\psi$, and thus

(51) $\quad \dot{u}>\exp \left\{-\kappa \int_{0}^{\psi} \frac{r^{2} \sin \psi}{\Delta^{2}} d \psi\right\}$

for sufficiently small $\psi$. Then immediately $\dot{u}>0$ and this inequality holds throughout $\mathcal{I}_{R}$ and $\mathcal{I}_{L}$. Then as $2 \Delta-\left(1-r^{2} / m^{2}\right) \sin \psi>\sin \psi\left(1+m^{2} / r^{2}\right)>0$ in $\mathcal{I}_{R}$ and $-2 \Delta-\left(m^{2} / r^{2}-1\right) \sin \psi<$ $-2 \kappa r u+\sin \psi<0$ in $\mathcal{I}_{L}$ we may conclude

(52) $\frac{d \dot{V}_{R}}{d \psi}<0$ and $\frac{d \dot{V}_{L}}{d \psi}<0$

in $\mathcal{I}_{R}$ and $\mathcal{I}_{L}$ respectively.

Suppose there is a positive value $\hat{\psi} \leq \pi$ at which $\dot{r}=0$. Label the smallest such value again by $\hat{\psi}$. Since $(52)$ holds on $0<\psi \leq \hat{\psi}$, and since $\dot{V}_{R}(0)=0$ we may deduce that $\dot{V}_{R}(\hat{\psi})<0$. But by (50), $\dot{u}(\hat{\psi})>0$, and then $\dot{V}_{R}(\hat{\psi})=\pi\left(r^{2}-m^{2}\right) \dot{u}(\hat{\psi})>0$. This contradiction establishes that $\dot{r}<0$ on $0<\psi \leq \pi$. Similarly, suppose there is a negative value $\hat{\psi} \geq-\pi$ at which $\dot{r}=0$. Label the largest such value again by $\hat{\psi}$. Since (52) holds on $\hat{\psi} \leq \psi<0$, and since $\dot{V}_{L}(0)=0$ we may deduce that $\dot{V}_{L}(\hat{\psi})<0$. But by $(50), \dot{u}(\hat{\psi})>0$, and $\dot{V}_{L}(\hat{\psi})=\pi\left(m^{2}-r^{2}\right) \dot{u}(\hat{\psi})>0$. This contradiction establishes that $\dot{r}>0$ on $0>\psi \geq-\pi$. We have proved

Lemma 3.5 On $-\pi \leq \psi<0, \dot{r}>0$, and on $0<\psi \leq \pi, \dot{r}<0$.

Theorem 3.6 For every $a$ and $b, 0<a<b<\infty$ and every $\psi_{a}, \psi_{b},-\pi \leq \psi_{a} \leq 0$ and $0 \leq \psi_{b} \leq \pi$, there is a solution of (4),(5) that attains inclination angles $\psi_{a}$ at $a$ and $\psi_{b}$ at $b$.

Proof. For $-\pi \leq \psi_{a} \leq 0$, there is a unique $\alpha_{0}=\alpha_{0}(h, m)$ such that $r\left(\psi_{a}\right)=\alpha_{0}$. Since $r(\psi) \leq m$ for $-\pi \leq \psi_{a} \leq 0$, we have $\alpha_{0}<m$. In fact, if $m=a$, then $\alpha_{0}<a$. By continuity, there is some $\bar{m}>a$ so that (keeping $h$ fixed) we have $\alpha_{0}(h, m)<a$ for every $m \in(a, \bar{m})$. Now, keeping $m \in(a, \bar{m})$ fixed and increasing $h$, we have $L \leq \alpha_{0}$ and $\lim _{h \rightarrow \infty} L=m$. Then $\partial \alpha_{0} / \partial h=\dot{r}>0$, implies that there is a unique $h=h(m)$ so that

$$
\alpha_{0}(h, m)=a .
$$

This relation holds for $h=h(m)$ and $m \in(a, \bar{m})$.

For $0 \leq \psi_{b} \leq \pi$, there is a unique $\beta_{0}=\beta_{0}(h, m)$ such that $r\left(\psi_{b}\right)=\beta_{0}$. Let

$$
\bar{m}=\sup \left\{\rho: \exists ! h=h(m)>0 \text { such that } \alpha_{0}(h, m)=a \text { for } a<m<\rho\right\} .
$$

Note that $\bar{m}$ may be infinite. The argument above shows that $\bar{m}>a$. If $m$ is close enough to $a$ then $h$ must be large enough so that $r\left(\psi_{a}\right)=\alpha_{0}$, and thus the fact that $h \rightarrow \infty$ implies $L \rightarrow m$ gives $\beta_{0}<b$. Thus, by continuity, it remains to show

$$
\beta_{0}(h(m), m)>b
$$

for some $m<\bar{m}$. If $\bar{m}=\infty$, or even $\bar{m}>b$, then clearly $\alpha_{0}(h(b), b)=a$ and $\beta_{0}(h(b), b)>b$ (as $\left.\beta_{0}(h, m)>m\right)$ and thus continuity implies $\beta_{0}(h(m), m)=b$ for some $m<b<\bar{m}$.

Claim: if $\bar{m}<\infty$, then $\lim _{m \rightarrow \bar{m}} h(m)=0$ and $\lim _{m \rightarrow \bar{m}} \beta_{0}(h(m), m)=\infty$.

First assume that $\limsup _{m \rightarrow \bar{m}} h(m)=h_{0}>0$, then $\alpha_{0}(h, m)<a$ for $0<h<h_{0}$ and $\alpha_{0}\left(h_{0}, \bar{m}\right)=$ $a$. Thus for $0<h<h_{0}$ we have $\alpha_{0}(h, \bar{m})<a$ and there is some $\epsilon_{0}>0$ for which $\alpha_{0}(h, \bar{m}+\epsilon)<a$ for $0<\epsilon<\epsilon_{0}$. Thus $h(\bar{m}+\epsilon)$ is well defines, which contradicts the definition of $\bar{m}$. The first part of the claim follows. 
If $\pi / 2<\psi \leq \pi$, then Lemma 3.4 implies the result. In the case that $0<\psi \leq \pi / 2$ the convergence is not uniform. However, from [1], and that $\psi=\psi(r)$ in this region, we have

$$
\frac{v\left(r, h_{1}\right)}{v\left(r, h_{2}\right)} \leq \frac{h_{1}}{h_{2}}
$$

for $0<h_{1}<h_{2}$. Note that $v(r, h)=\sin (\psi(r ; h))$. Thus, taking the limit as $h_{1} \rightarrow 0$ implies $\sin (\psi(r)) \rightarrow 0$. Thus $r(\psi) \rightarrow \infty$. and the second part of the claim follows.

Thus, if $\bar{m}<\infty$, continuity implies $\beta_{0}(h(m), m)=b$ for some $m<\bar{m}$.

Remark 3.7 We have actually proven more. First observe that this also implies existence for the "opposite" inclination angles, by reflection: $\psi_{a} \in[0, \pi]$ and $\psi_{b} \in[-\pi, 0]$. More subtly, there are solution meridians with $\psi_{a}, \psi_{b} \in[-\pi, 0]$ or $\psi_{a}, \psi_{b} \in[0, \pi]$ that may be extended to the $(m, h)$ point where $\psi=0$. Clearly, for these meridians, the above method gives existence. What is missing is a handy way of knowing if given boundary conditions are giving such a solution meridian.

\section{State of the problem}

Figure 8 is a contact angle diagram where the shaded regions represent the cases where existence has been proven. In the region $(-\pi / 2, \pi / 2) \times(-\pi / 2, \pi / 2)$ existence was proven in [1], and uniqueness there follows from general comparison principles: see [4]. The current paper shows existence both for $[-\pi / 2, \pi / 2] \times[-\pi / 2, \pi / 2]$ as well as $[-\pi, 0] \times[0, \pi]$ and $[0, \pi] \times[-\pi, 0]$. For many applications proof of the following is needed:

Conjecture 4.1 Given $0<a<b<\infty$ and inclination angles $\psi_{a}$ and $\psi_{b}$ in $[-\pi, \pi]$ there exists a unique embedded solution to (6),(7),(8) so that $\psi=\psi_{a}$ when $r=a$ and $\psi=\psi_{b}$ when $r=b$.

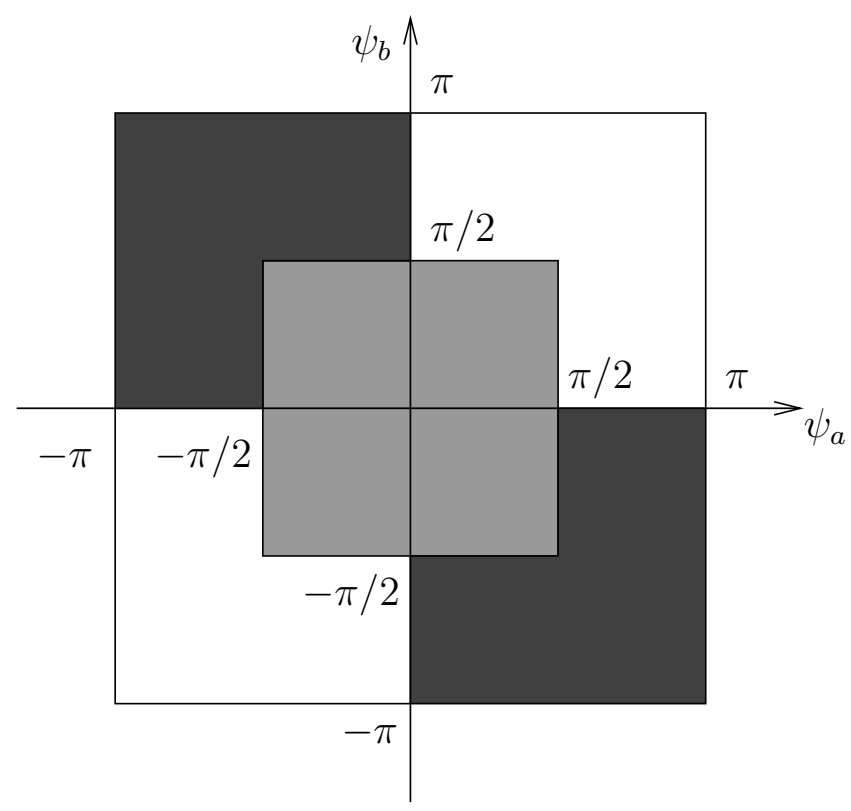

Figure 8: Contact angle diagram.

The author would like to thank Alan Elcrat, Henry Wente and the referee, who all made helpful comments. Also, portions of this work were completed while the author was both at Wichita State University and The University of Toledo. 


\section{References}

[1] Alan Elcrat, Tae-Eun Kim, and Ray Treinen, Annular capillary surfaces, Arch. Math. (Basel) 82 (2004), no. 5, 449-467.

[2] Alan Elcrat, Robert Neel, and David Siegel, Equilibrium configurations for a floating drop, J. Math. Fluid Mech. 6 (2004), no. 4, 405-429.

[3] Alan Elcrat and Ray Treinen, Numerical results for floating drops, Discrete Contin. Dyn. Syst. (2005), no. suppl., 241-249.

[4] Robert Finn, Equilibrium capillary surfaces, Grundlehren der Mathematischen Wissenschaften [Fundamental Principles of Mathematical Sciences], vol. 284, Springer-Verlag, New York, 1986.MR816345 (88f:49001)

[5] W. E. Johnson and L. M. Perko, Interior and exterior boundary value problems from the theory of the capillary tube, Arch. Rational Mech. Anal. 29 (1968), 125-143.

[6] John McCuan and Ray Treinen, Capillarity and Archimedes' principle of floatation, To Appear.

[7] David Siegel, Height estimates for capillary surfaces, Pacific J. Math. 88 (1980), no. 2, 471-515.

[8] Ray Treinen, A study of floating drops, Wichita State Univ., 2004.

[9] Bruce Turkington, Height estimates for exterior problems of capillarity type, Pacific J. Math. 88 (1980), no. $2,517-540$.

[10] Thomas I. Vogel, Symmetric unbounded liquid bridges, Pacific J. Math. 103 (1982), no. 1, 205-241. 